\title{
Muscle-specific changes in length-force characteristics of the calf muscles in the spastic Han-Wistar rat
}

\author{
Annesofie T. Olesen, ${ }^{1,2,3}$ Bente R. Jensen, ${ }^{1}$ Toni L. Uhlendorf, ${ }^{4}$ Randy W. Cohen, ${ }^{4}$ Guus C. Baan, ${ }^{2}$ \\ and Huub Maas ${ }^{2}$ \\ ${ }^{1}$ Department of Nutrition, Exercise and Sports, Faculty of Science, University of Copenhagen, Copenhagen, Denmark; \\ ${ }^{2}$ MOVE Research Institute Amsterdam, Faculty of Human Movement Sciences, VU University, Amsterdam, The Netherlands; \\ ${ }^{3}$ Institute of Sports Medicine Copenhagen, Bispebjerg Hospital, Copenhagen, Denmark; and ${ }^{4}$ Department of Biology, \\ California State University, Northridge, California
}

Submitted 1 July 2014; accepted in final form 28 August 2014

\begin{abstract}
Olesen AT, Jensen BR, Uhlendorf TL, Cohen RW, Baan GC, Maas H. Muscle-specific changes in length-force characteristics of the calf muscles in the spastic Han-Wistar rat. J Appl Physiol 117: 989-997, 2014. First published September 4, 2014; doi:10.1152/japplphysiol.00587.2014.-The purpose of the present study was to investigate muscle mechanical properties and mechanical interaction between muscles in the lower hindlimb of the spastic mutant rat. Length-force characteristics of gastrocnemius (GA), soleus (SO), and plantaris (PL) were assessed in anesthetized spastic and normally developed Han-Wistar rats. In addition, the extent of epimuscular myofascial force transmission between synergistic GA, SO, and PL, as well as between the calf muscles and antagonistic tibialis anterior (TA), was investigated. Active length-force curves of spastic GA and PL were narrower with a reduced maximal active force. In contrast, active length-force characteristics of spastic SO were similar to those of controls. In reference position $\left(90^{\circ}\right.$ ankle and knee angle), higher resistance to ankle dorsiflexion and increased passive stiffness was found for the spastic calf muscle group. At optimum length, passive stiffness and passive force of spastic GA were decreased, whereas those of spastic SO were increased. No mechanical interaction between the calf muscles and TA was found. As GA was lengthened, force from SO and PL declined despite a constant muscle-tendon unit length of SO and PL. However, the extent of this interaction was not different in spastic rats. In conclusion, the effects of spasticity on length-force characteristics were muscle specific. The changes observed for GA and PL muscles are consistent with the changes in limb mechanics reported for human patients. Our results indicate that altered mechanics in spastic rats cannot be attributed to differences in mechanical interaction, but originate from individual muscular structures.
\end{abstract}

muscle spasticity; mechanical properties; myofascial force transmission; muscle stiffness

MUSCLE SPASTICITY HAS BEEN defined as a "velocity-depended resistance to stretch" (24) and arises secondary to upper motoneuron lesions with cerebral palsy and stroke as the most common examples (12). Individuals suffering from spasticity typically experience muscle weakness, enhanced joint stiffness, increased muscle tone, reduced range of joint motion, increased antagonistic co-contraction, and exaggerated reflexes $(1,3,6,12,13,32,41,48)$. These effects severely impair the ability to perform daily activities, and treatment is often needed.

To asses whether some of these symptoms are related to altered mechanical properties of muscle-tendon units (MTUs),

\footnotetext{
Address for reprint requests and other correspondence: H. Maas, MOVE Research Institute Amsterdam, VU Univ. Amsterdam, Van der Boerchorststraat 7, 1081 BT Amsterdam, The Netherlands (e-mail: h.maas@vu.nl).
}

passive and active length-force characteristics have been estimated as joint moment as a function of gastrocnemius (GA) fascicle length. Spastic GA was found to be stiffer than normally developed in such studies $(2,3)$. The active lengthforce curve of the spastic GA was narrower and the optimum length was shifted to a shorter fascicle length than in normally developed GA $(2,15,16)$. However, estimating length-force characteristics from joint moments can be misleading because this is the net effect of forces exerted by several MTUs and passive structures around the joint, which depend on their moment arms $(11,30)$. Therefore, it remains unclear whether these findings can be attributed to individual MTUs.

Muscle fiber force can be transmitted to bone via the tendon or aponeurosis, termed myotendinous force transmission. Force can also be transmitted via intermuscular and extramuscular connective tissues (29), termed epimuscular myofascial force transmission. Mechanical interaction between muscles is an effect of such force transmission, which is defined as a change in force of restrained muscles caused by changes in MTU length of an adjacent muscle (28). The degree of force transmission between muscles depends on the force-transmitting ability of the myofascial pathways. Stiffer pathways will transmit relatively more force than less stiff pathways. Due to strong indications of stiffer connective tissues in spastic muscles $(5,43)$, it is likely that epimuscular myofascial pathways in spastic limbs are stiffer and will transmit relatively more force. Effects of epimuscular myofascial force transmission have been found in the forearm of human patients with spastic cerebral palsy $(10,42)$. These measurements were not performed in normally developed limbs. Therefore, it is not known whether effects of epimuscular myofascial force transmission are enhanced in spastic limbs.

A spastic Han-Wistar mutant rat model was first described in 1976 (37). It was reported to exhibit progressive spastic paresis, hindlimb rigidity, forelimb tremor, hyperactivity, and ataxia. Note also that in human patients suffering from spasticity, reduced range of joint motion is a frequently observed symptom (see above). In these rats, hyperactivity and tremor are initiated first at the age of 25-30 days. From the 35th day, ataxia becomes more progressive in both forelimbs and hindlimbs. At 42-50 days postnatal, spastic paresis is manifested by limb rigidity and more plantar-flexed paws (37), which is also typical in humans with spastic lower legs. The spastic mutant rat has previously been used for neurophysiological studies $(7,8,39,44)$, but the mechanical properties of its skeletal muscles have never been investigated. 
The first aim of the present study was to investigate passive and active length-force characteristics of the calf muscles in the spastic mutant rat. We hypothesized that 1 ) the length-force characteristics are narrower and shifted to a more plantarflexed position of the ankle; 2) maximal active force is reduced; and 3) passive muscle stiffness is increased as suggested for human patients suffering from spasticity. The second aim of our study was to investigate whether mechanical interaction between hindlimb synergistic and antagonistic muscles is affected by spasticity. We hypothesized that the extent of such interaction between muscles is higher in spastic limbs than in normally developed limbs.

\section{METHODS}

Animals. Ten Han-Wistar rats (6 females, 4 males, weight $160 \pm$ $30 \mathrm{~g}$, age $58 \pm 6$ days, Table 1) suffering from a neurodegenerative disorder characterized by spasticity (37) were used in this study (SP rats). The SP rats were obtained from the California State University, Northridge breeding colony. As control rats, eight normally developed Han-Wistar rats (4 females, 4 males, weight $180 \pm 27 \mathrm{~g}$, estimated age 7-9 wk based on the Charles River growth curve; Table 1) were used: two littermates of the SP rats and six rats from Charles River (Germany). At the time of testing, the SP rats exhibited crooked front paws and rigidity in the hindlimbs in different degrees. The rigidity caused the rats to walk on tiptoes with a more plantar-flexed ankle position than controls (Supplementary Materials, compare Video 1 and Video 2). In severe cases, the rats were not able to stand on their legs, but lay with fully extended knees and plantar-flexed ankles when resting. When initiating locomotion, voluntary ankle dorsiflexion seemed difficult and, in a single case, was not possible (Supplementary Materials, Video 3). Surgical and experimental procedures were in strict agreement with the guidelines and regulations concerning animal welfare and experimentation set forth by Dutch law and were approved by the Committee on Ethics of Animal Experimentation at the VU University, Amsterdam (permit FBW 10-04).

Surgical procedures. According to standard procedures in our laboratory [e.g., (26)], rats were deeply anesthetized by intraperitoneally injecting urethane (initial dose $1.2 \mathrm{ml} / 100 \mathrm{~g}$ body mass administered by two injections with 10-min intervals). If paw withdrawal reflex was present $15 \mathrm{~min}$ or longer after the initial dose, another 0.2 $\mathrm{ml}$ of urethane was injected. Ambient temperature and air humidity were kept constant at $22 \pm 0.5^{\circ} \mathrm{C}$ and $70 \pm 10 \%$, respectively, by a computer-controlled air conditioning system (Holland Heating, Waalwijk, The Netherlands). Body temperature was monitored and main-

Table 1. Descriptive and anatomical data

\begin{tabular}{lcc}
\hline \hline & SP, $n=10$ & Controls, $n=8$ \\
\hline Gender, male/female & $4 / 6$ & $4 / 4$ \\
Age, days* & $58 \pm 6$ & $49-63 \dagger$ \\
Body mass, g* & $161 \pm 30$ & $180 \pm 27$ \\
Lower leg length, mm & $38.6 \pm 2.4$ & $38.4 \pm 2.9$ \\
GA muscle belly length at $\mathrm{L}_{\text {ref }}, \mathrm{mm}$ & $25.6 \pm 1.6$ & $25.1 \pm 1.8$ \\
GA tendon length at $\mathrm{L}_{\text {ref }}, \mathrm{mm}$ & $9.6 \pm 1.2$ & $9.7 \pm 1.4$ \\
GA MTU length at $\mathrm{L}_{\text {ref }}, \mathrm{mm}$ & $35.2 \pm 2.3$ & $34.9 \pm 2.2$ \\
GA mass, g & $0.80 \pm 0.19$ & $0.93 \pm 0.10$ \\
SO mass, g & $0.07 \pm 0.01$ & $0.07 \pm 0.01$
\end{tabular}

GA, gastrocnemius; $\mathrm{L}_{\text {ref }}$, reference length; MTU, muscle-tendon unit; SO, soleus; SP, spastic. *Days of age and body mass at testing day. Length of lower leg and length of GA muscle belly, tendon, and MTU were estimated from photographs of the experimental leg with MTUs at $\mathrm{L}_{\text {ref }}$ and muscles relaxed. $\dagger$ Ages of control rats were estimated from growth curves and were therefore not statistically compared. No statistical difference between groups was found $(P>0.05)$. Values are means $\pm \mathrm{SD}$. tained at $\sim 37^{\circ} \mathrm{C}$ using an electrical heating pad. To prevent dehydration, exposed tissues were regularly irrigated with isotonic saline.

Before cutting the tendons from the skeleton, markers (knot with 7-0 suture; Prolene, Ethicon) were placed on the distal tendons of GA, soleus (SO), plantaris (PL), tibialis anterior (TA), and peroneus longus tendon to mark the position that corresponds to $90^{\circ}$ ankle and knee angles (Fig. 1A). This position will be referred to as reference length $\left(\mathrm{L}_{\mathrm{ref}}\right)$. Skin and biceps femoris were removed from the left hindlimb. The TA tendon distal to the retinaculum and a piece of the medial cuneiform bone with the TA tendon insertion were dissected free. The retinaculum at the ankle was left intact. The distal PL tendon was dissected free and severed distally to the calcaneus. The distal SO tendon was dissected free and severed from the Achilles tendon. The tendons of TA, SO, and PL were each connected to force transducers (maximal load $25 \mathrm{~N}$, error $<0.1 \%$, compliance of $0.0162 \mathrm{~mm} / \mathrm{N}$; BLH Electronica, Canton, MA) via a Kevlar thread (4\% elongation at break load of $800 \mathrm{~N}$; Goodfellow, Cambridge, UK). The distal tendon of GA, still attached to a cut piece of calcaneus, was connected to a different force transducer (maximal load $50 \mathrm{~N}$, error $<0.1 \%$, compliance of $0.0048 \mathrm{~mm} / \mathrm{N}$; Hottinger Baldwin) via a Kevlar thread. The biceps femoris muscle, the distal tendons of GA, SO, PL, and TA, a part of the medial cuneiform bone, and a part of the calcaneus bone were the only structures separated from the otherwise intact lower leg. All connective tissues between and around muscle bellies were left intact. The lateral side of the femur was exposed by dissection between quadriceps femoris and the hamstring muscles, and a metal clamp was attached. The foot was attached to a plastic plate using 1-0 silk suture and tie-wraps. The proximal end of the lateral GA was marked with a pen, and the distal end of the GA muscle belly was marked with piece of black thread sutured to the surface (7-0 suture; Prolene, Ethicon).

The common peroneal nerve was cut just distally to its branching from the sciatic nerve. Custom-made bipolar cuff electrodes were placed on the common peroneal nerve and on the sciatic nerve. Stimulation of the common peroneal nerve excited the ankle dorsiflexors, including TA and the peroneal muscles. Stimulation of the sciatic nerve excited the ankle plantar flexors, including GA, SO, and PL (Fig. 1).

Experimental setup. The rat was placed prone on a horizontal heating pad. The femur and foot were secured vertically such that the knee and ankle angle were $90^{\circ}$ (Fig. 1A). Force transducers were placed in the line of pull of the muscles. Due to space limitations, the Kevlar threads of TA and PL muscles were connected to their force transducers via low-friction pulleys (26). Muscles were excited by electrical stimulation of the sciatic nerve and the severed common peroneal nerve. Stimulation was supramaximal and consisted of two single twitches followed by a $100-\mathrm{Hz}$ pulse train of $500 \mathrm{~ms}$ (square pulses, amplitude $0.3-0.5 \mathrm{~mA}$, pulse width $100 \mu \mathrm{s}$ ) from a constant current source (DS3 Constant Current Isolated Stimulator; Digitimer, Hertfordshire, UK). The two twitches were evoked to enable the muscles to adapt to the imposed length and relative position (46). Force data were collected at $1 \mathrm{kHz}$ using a custom-built constant current strain gauge amplifier $(400-1,600 \times)$ and an A/D converter (PCI-6221; National Instruments, Austin, TX). A custom-made data acquisition program using LabVIEW (version 9.0.1f1) was used to control stimulation of the nerves and for data collection and storage.

Experimental protocols. Initially, to prevent history effects of muscle contractions at high lengths, the three calf muscles (i.e., GA, PL, and SO) were excited at low and high lengths repeatedly until force output at low length was reproducible for each muscle (19). TA muscle was excited repeatedly at $\mathrm{L}_{\text {ref }}$, because TA was kept at $\mathrm{L}_{\text {ref }}$ throughout all experimental protocols.

Isometric forces exerted at the distal tendons of all four muscles were measured for two protocols (see Fig. 1, $B$ and $C$ ): 1) Increasing the length of the three calf muscles ( $l_{\text {calf }}$ MTU $)$ simultaneously from the active slack length of GA to $2 \mathrm{~mm}$ above active optimum length of GA. The muscles were lengthened distally in steps of $1 \mathrm{~mm}$. TA was kept at $\mathrm{L}_{\text {ref. }}$ Common peroneal and sciatic nerves were stimulated 


\section{A Experimental setup}

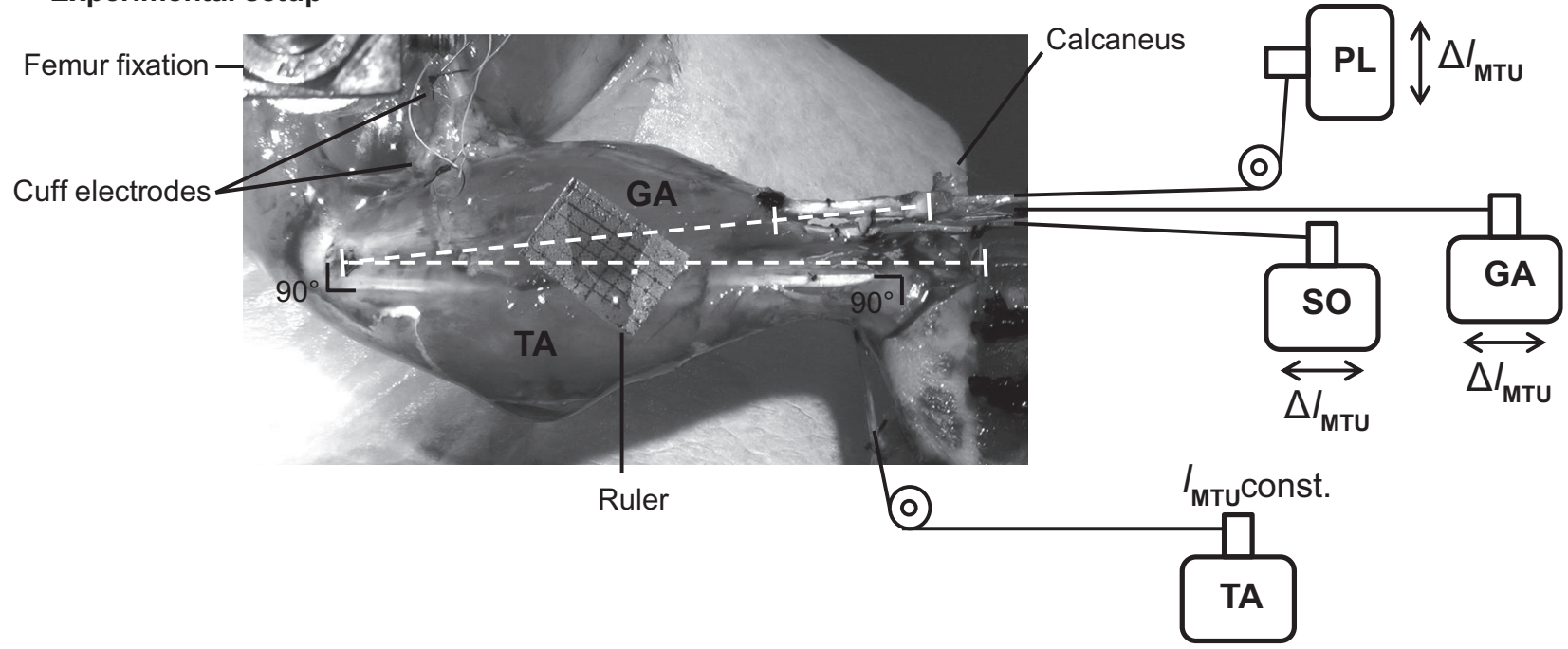

B Protocol 1

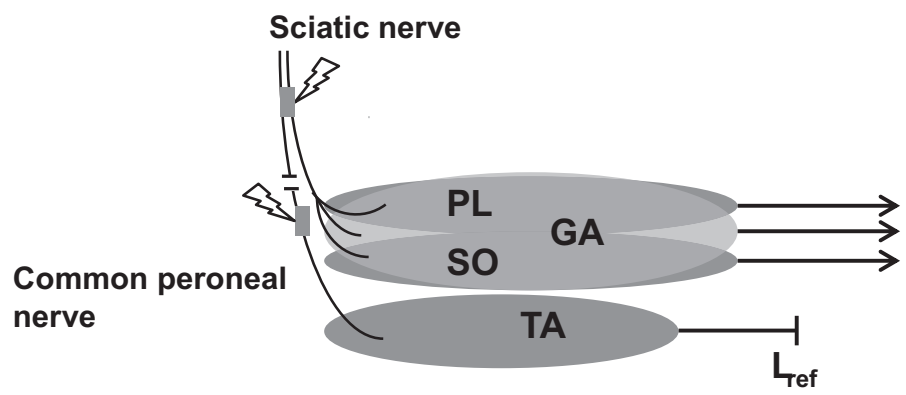

C Protocol 2

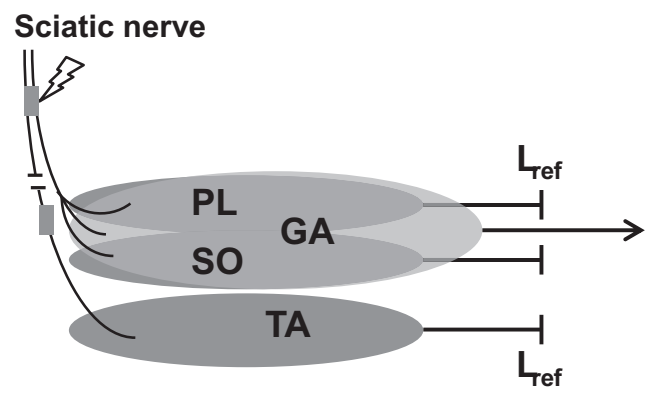

Fig. 1. Experimental setup and protocols. A: lateral view of the left rat hindlimb with anterior and posterior crural compartments exposed, and the experimental setup. The femur was fixed vertically with a metal clamp and the foot was fixed vertically to a plastic plate. Knee and ankle angles were kept at $90^{\circ}$, and the lower leg was held horizontal. Tendons of gastrocnemius (GA), plantaris (PL), and soleus (SO) muscles were connected to force transducers with adjustable positions $\left(\Delta l_{\mathrm{MTU}}\right)$. The tibialis anterior (TA) tendon was connected to a force transducer and kept at reference length in all experimental conditions. Markers were used to set the reference lengths $\left(\mathrm{L}_{\mathrm{ref}}\right)$. Cuff electrodes were mounted on the sciatic and common peroneal nerves. Dashed lines indicate lengths of lower leg and GA muscle belly, tendon, and muscle-tendon unit (MTU) (for details see METHODS). B: protocol 1; the lengths of all calf MTU were increased simultaneously at the distal end from the active slack length of GA to $2 \mathrm{~mm}$ above active optimum length of GA. TA was kept at reference length ( $\mathrm{L}_{\text {ref }}$ ). Common peroneal and sciatic nerves were stimulated simultaneously. C: protocol 2; the length of GA was increased exclusively from its active slack length to $2 \mathrm{~mm}$ above optimum length. SO, PL, and TA muscles were kept at their $\mathrm{L}_{\text {ref. }}$ Only the sciatic nerve was stimulated.

simultaneously. The purposes of protocol 1 were $a$ ) to assess the length-force characteristics of the calf muscles, both individually and as a group; and $b$ ) to quantify the extent of mechanical interaction between the calf muscles and the antagonistic TA. By restraining TA, the changes in force output from TA must be attributed to effects of epimuscular myofascial force transmission between the calf muscles and TA. 2) Increasing the length of GA exclusively ( $\left.l_{\mathrm{GA}} \mathrm{MTU}\right)$ from its active slack length to $2 \mathrm{~mm}$ above optimum length in steps of $1 \mathrm{~mm}$. $\mathrm{SO}, \mathrm{PL}$, and TA muscles were kept at $\mathrm{L}_{\text {ref. }}$ The purpose of protocol 2 was to quantify the extent of mechanical interaction between GA and the synergistic SO and PL muscles. Therefore, in this protocol only the sciatic nerve was stimulated. After each contraction the muscles were released to passive slack length and rested for 2 min to minimize muscle fatigue. Before and after both protocols, the muscles were excited at lengths near optimum length to monitor any change in muscle conditions (e.g., fatigue). After protocol 1, total GA force was decreased by less than 12\%. Protocol 2 resulted in an additional decrease of GA force of less than $5 \%$.

Digital still pictures (Panasonic Camcorder HC-V720) were taken of the experimental leg from a lateral view in passive condition at $\mathrm{L}_{\text {ref }}$ in protocol 1. After the mechanical measurements were completed, lateral and medial GA and SO were harvested to obtain muscle masses (Table 1).

Data analysis. Forces exerted at the different tendons of the calf muscles were analyzed individually, but also as the sum indicated as force of the calf muscle group. Passive and active force was extracted from the measured force as follows: passive force was calculated in Matlab 2013b as the mean of the total force output for $50 \mathrm{~ms}$ between the second twitch and the tetanic stimulation train. Total force during the tetanus was calculated as the mean of the total force output of the last $50 \mathrm{~ms}$ of the pulse train. Active force was assessed by subtracting passive force from total force at equal MTU length. Active force as a function of MTU length was fitted to a polynomial of maximal sixth order using stepwise regression $\left(R^{2}>0.99\right.$, mean $\left.R^{2}=1.00\right)(20,26)$. Considering the high number of data points used (on average $15.3 \pm$ 1.5 , minimally 13 ), this maximal order was deemed appropriate. Maximal active force, active slack $\left(\mathrm{L}_{\text {slack }}\right)$, and active optimum length $\left(\mathrm{L}_{\mathrm{opt}}\right)$ were determined from the fitted curves. The length range of the ascending part of the active length-force curve was calculated as the length range between active slack length and optimum length. Passive forces as a function of MTU length were fitted to a double exponential function as $\mathrm{f}(\mathrm{x})=\mathrm{a} \cdot \mathrm{e}^{\mathrm{b} \cdot \mathrm{x}}+\mathrm{c} \cdot \mathrm{e}^{\mathrm{d} \cdot \mathrm{x}}\left(R^{2}>0.99\right.$, mean $\left.R^{2}=1.00\right)(9)$. 
Passive force at $\mathrm{L}_{\text {ref }}$ and at $\mathrm{L}_{\text {opt }}$ was determined from the fitted curves. Passive stiffness of the MTU, calculated as the slope of the passive length-force curve, was assessed at $\mathrm{L}_{\text {ref }}$ and $\mathrm{L}_{\text {opt }}$.

To assess the extent of mechanical interactions between synergistic and antagonistic muscles, total forces exerted at the distal tendon of the length-restrained muscles (TA in protocol 1; SO and PL in protocol 2) were used. Note that passive forces were very small in those conditions $(<0.05 \mathrm{~N}$ in TA, $<0.04 \mathrm{~N}$ in SO, and $<0.04 \mathrm{~N}$ in PL). Total forces were fitted to a third-order polynomial $\left(R^{2}>0.62\right.$, mean $R^{2}=0.92$ ). Total force was normalized to the total force of the respective muscle when all muscles were aligned at $\mathrm{L}_{\text {ref. }}$. Length was normalized to the length range of the ascending part of the active length-force curve of GA.

The length of GA muscle belly and MTU in passive condition at $\mathrm{L}_{\text {ref }}$ (protocol 1) were measured from digital still pictures (4.14 megapixels, $0.057 \pm 0.002 \mathrm{~mm}$ per pixel). Tendon length was subsequently calculated by subtracting muscle belly length from MTU length. From the same pictures, length of the lower leg was estimated as the perpendicular distance from the origin of lateral GA to the surface of the footplate.

Statistics. All values are presented as mean \pm SD. Statistical analysis was performed in SPSS 20. Group differences in length-force curves were tested with a two-way ANOVA (MTU length $\times$ group) with MTU length as the repeated measurements factor. Because sphericity was violated consistently, Greenhouse-Geisser correction was applied in all ANOVAs. $t$-tests were used for single parameter comparison, and unequal variance in the two groups was assumed. When the ANOVAs indicated a significant group effect or interaction between factors (group and length), forces and stiffnesses were compared at reference length. Parameters related to slack and optimum length were tested in all cases, because these were not included in the ANOVAs. Gender distribution was $\chi^{2}$ tested. Ages were not statistically compared because the ages of the control rats were estimated (see above). Level of significance was set at $P<0.05$.

\section{RESULTS}

Descriptive and anatomical parameters. No significant differences were found for the anatomical parameters: body mass; lower leg length; GA mass; SO mass; and lengths of GA muscle belly, MTU, and tendon at reference length corresponding to an ankle and knee joint angle of $90^{\circ}$ (Table 1). Neither did gender distribution differ between groups.

Length-force characteristics of the calf muscle group. The passive and active length-force characteristics of the group of calf muscles (i.e., the force of GA, PL, and SO summed at each MTU length) were significantly affected by spasticity (Fig. $2 A)$. For both passive and active forces, ANOVA indicated group ( $P=0.038$ and $P<0.001$, respectively) and MTU length effects (both $P<0.001$ ), as well as interaction between these factors $(P=0.032$ and $P<0.001$, respectively). This interaction indicates that when MTU length changed, force responded differently in the two groups, indicative of the curves having different slopes. Maximal active force of the calf muscles was $31 \%$ lower in the SP group $(11.6 \pm 2.3 \mathrm{~N})$ than in the control group $(16.8 \pm 2.9 \mathrm{~N}, P<0.001$, Fig. $2 B)$. No difference was found in active force at $\mathrm{L}_{\text {ref }}($ Fig. $2 A)$. For the SP group, optimum length was located at an MTU length closer to $\mathrm{L}_{\text {ref }}$ than for the control group $(P=0.005$, Table 2$)$. However, active slack length relative to $\mathrm{L}_{\text {ref }}$ was not significantly different between groups. Consequently, the length range of the ascending part of the active length-force curve for the calf muscles of the SP group was shorter than that of controls $(P=0.030$, Table 2$)$. At $\mathrm{L}_{\mathrm{ref}}$, both passive force and

\section{A Length-force curves of the calf muscles}
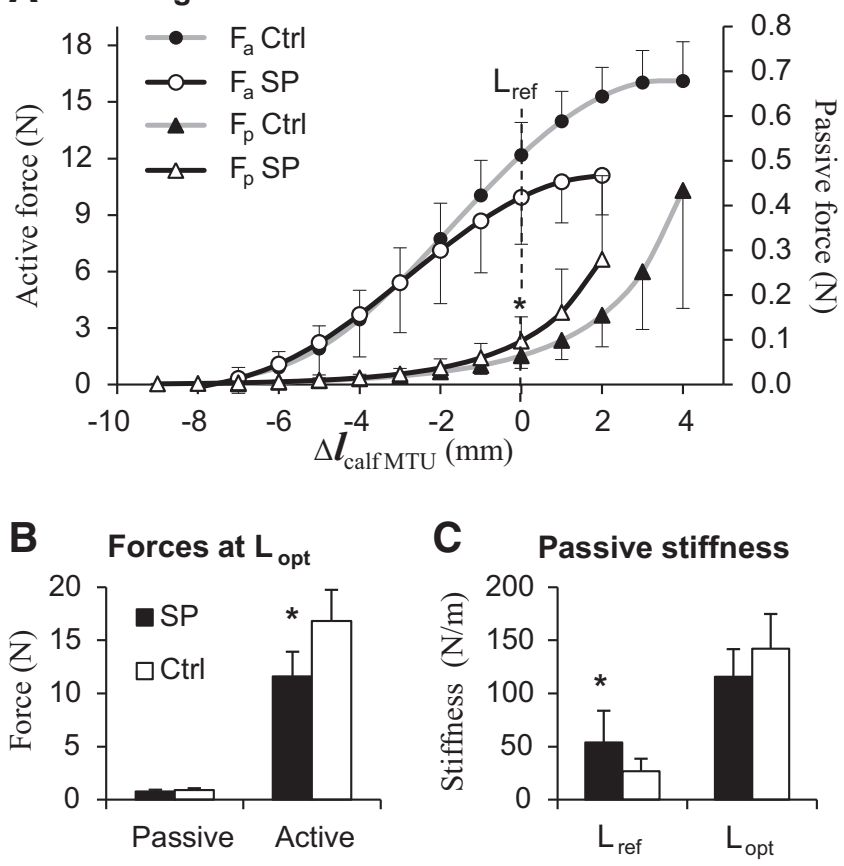

Fig. 2. Length-force characteristics of the calf muscle group (the sum of GA, $\mathrm{SO}$, and PL forces). $A$ : active and passive length-force curves $\left(\mathrm{F}_{\mathrm{a}}\right.$ and $\mathrm{F}_{\mathrm{p}}$, respectively) of the calf muscles relative to reference length ( $\mathrm{L}_{\mathrm{ref}}=0 \mathrm{~mm}$ ). Group (active $P<0.001$, passive $P=0.038)$ and length $(P<0.001)$ effects were found, as was interaction (group $\times$ length, active $P<0.001$, passive $P=0.032)$. The passive force at $\mathrm{L}_{\mathrm{ref}}$ was higher in spastic rats than in control rats $(* P=0.038)$. The active force at $\mathrm{L}_{\text {ref }}$ was not affected by spasticity. $B$ : active and passive forces of the calf muscles at optimum length $\left(\mathrm{L}_{\mathrm{opt}}\right)$. The active force at $\mathrm{L}_{\text {opt }}$ is per definition the maximal active force. $C$ : passive stiffness of the calf muscles at $\mathrm{L}_{\mathrm{ref}}$ and $\mathrm{L}_{\text {opt }}{ }^{*}$ Significant difference $(P<0.05)$. Values are means $\pm \mathrm{SD}$.

MTU stiffness in the SP group were $83 \%$ and $100 \%$ higher, respectively, than in the controls $(P=0.038$ and $P=0.030$, respectively; Fig. 2, $A$ and $C$ ). Passive force and stiffness at $\mathrm{L}_{\text {opt }}$ in the SP group were not different from those in controls (Fig. 2, $B$ and $C$ ). These results indicate that the active length-force curve of the calf muscles in the SP group was narrower and its maximal active force was substantially lower. At the reference position of the ankle and knee joint, the calf muscles in the SP group were operating closer to optimum length, and the passive stiffness was higher.

Length-force characteristics of GA. Passive and active length-force characteristics of GA, lengthened simultaneously with PL and SO (protocol 1), were significantly affected by spasticity (Fig. 3A). For both passive and active GA forces, ANOVA indicated a MTU length effect $(P<0.001)$ and interaction between the factors group and MTU length $(P=$ 0.045 and 0.002 , respectively), but no group effect. Maximal active force of GA was $31 \%$ lower in the SP group (8.80 \pm $1.80 \mathrm{~N})$ than in the control group $(12.70 \pm 2.38 \mathrm{~N}, P=0.001$, Fig. $4 A$ ). Note that in both groups, GA muscle exerted $\sim 75 \%$ of the maximal active force of the calf muscles. No difference was found in active force at $\mathrm{L}_{\text {ref }}$ (Fig. 3A). The optimum length of GA was at an MTU length closer to $\mathrm{L}_{\text {ref }}$ in the SP group than in the control group $(P=0.006$, Table 2$)$. No difference was found in active slack length relative to $\mathrm{L}_{\text {ref. }}$ Similar to the calf muscles, the length range of the ascending part of the active 
Table 2. Slack and optimum lengths

\begin{tabular}{lrrc}
\hline \hline & $\mathrm{SP}, n=10$ & Controls, $n=8$ & $P$ \\
\hline Calf muscles & & & \\
$\quad \mathrm{L}_{\text {slack}}, \mathrm{mm}$ & $-8.5 \pm 1.8$ & $-8.2 \pm 1.3$ & N.S. \\
$\mathrm{L}_{\text {opt }}, \mathrm{mm}$ & $1.7 \pm 1.2^{*}$ & $3.7 \pm 1.5$ & 0.005 \\
$\mathrm{~L}_{\text {asc }}, \mathrm{mm}$ & $10.1 \pm 1.3^{*}$ & $11.8 \pm 1.8$ & 0.030 \\
$\mathrm{GA}$ & & & \\
$\quad \mathrm{L}_{\text {slack}}, \mathrm{mm}$ & $-8.2 \pm 1.7$ & $-8.1 \pm 1.3$ & N.S. \\
$\mathrm{L}_{\text {opt }}, \mathrm{mm}$ & $2.0 \pm 1.1^{*}$ & $4.1 \pm 1.6$ & 0.006 \\
$\mathrm{~L}_{\text {asc }}, \mathrm{mm}$ & $10.2 \pm 1.1^{*}$ & $12.2 \pm 1.9$ & 0.014 \\
$\mathrm{PL}$ & & & \\
$\mathrm{L}_{\text {slack}}, \mathrm{mm}$ & $-7.7 \pm 1.9$ & $-7.7 \pm 1.6$ & N.S. \\
$\mathrm{L}_{\text {opt }}, \mathrm{mm}$ & $0.7 \pm 0.9^{*}$ & $2.5 \pm 1.2$ & 0.003 \\
$\mathrm{~L}_{\text {asc }}, \mathrm{mm}$ & $8.4 \pm 1.6^{*}$ & $10.2 \pm 1.1$ & 0.011 \\
$\mathrm{SO}$ & & & \\
$\mathrm{L}_{\text {slack}}, \mathrm{mm}$ & $-5.5 \pm 1.2$ & $-5.1 \pm 0.9$ & N.S. \\
$\mathrm{L}_{\text {opt }}, \mathrm{mm}$ & $2.1 \pm 1.1$ & $3.2 \pm 1.5$ & N.S. \\
$\mathrm{L}_{\text {asc }}, \mathrm{mm}$ & $7.6 \pm 1.3$ & $8.3 \pm 1.2$ & N.S. \\
\hline
\end{tabular}

Table 2. Slack length $\left(\mathrm{L}_{\text {slack }}\right)$ and optimum length $\left(\mathrm{L}_{\mathrm{opt}}\right)$ were measured relative to reference length. The ascending part of the active length-force curve $\left(\mathrm{L}_{\text {asc }}\right)$ was calculated as the length range between $\mathrm{L}_{\text {slack }}$ and $\mathrm{L}_{\text {opt. }}$. *Significant difference $(P<0.05)$. N.S., not significant $(P \geq 0.05)$. Values are means $\pm \mathrm{SD}$.

length-force curve for GA was shorter in the SP group than in controls $(P=0.014$, Table 2$)$. At $\mathrm{L}_{\text {ref }}$, passive force was not affected by spasticity (Fig. 3A), but passive MTU stiffness was higher in the SP group $(35.3 \pm 17.9 \mathrm{~N} / \mathrm{m})$ than in the control group $(19.8 \pm 8.7 \mathrm{~N} / \mathrm{m}, P=0.040$, Fig. $5 A)$. GA passive force and MTU stiffness at $\mathrm{L}_{\mathrm{opt}}$ was $24 \%$ and $26 \%$ lower, respectively, in the SP group than in controls $(P=0.014$ and 0.006 , respectively; Fig. $4 B$ and $5 B$ ). These data indicate that GA was the dominating muscle of the calf muscles. Accordingly, spasticity affected its length-force characteristics in a similar fashion as the calf muscle group.

Length-force characteristics of PL. The passive and active length-force characteristics for PL in protocol 1 of the SP group were significantly different from those of controls (Fig. $3 B$ ). For active PL force, ANOVA indicated an MTU length effect $(P<0.001)$ and interaction between groups and MTU length $(P<0.001)$, but no group effect. Maximal active force of PL was $41 \%$ lower in the SP group $(1.92 \pm 0.59 \mathrm{~N})$ than in the control group $(3.28 \pm 0.65 \mathrm{~N}, P<0.001$, Fig. $4 A)$. No difference was found in active force at $\mathrm{L}_{\text {ref }}$ (Fig. $3 B$ ). Optimum length of PL was located at a shorter MTU length relative to $\mathrm{L}_{\text {ref }}$ in the SP group than in controls $(P=0.003$, Table 2$)$. Because active slack length relative to $L_{\text {ref }}$ was not different, the length range of the ascending part of the active length-force curve for PL was also shorter in the SP group than in controls $(P=0.011$, Table 2$)$. For passive PL force, ANOVA indicated group $(P=0.023)$ and MTU length effects $(P<0.001)$, as well as interaction between these factors $(P=0.028)$. The PL passive force and MTU stiffness at $\mathrm{L}_{\text {ref }}$ was higher in the SP group than in the control group $(P=0.026$ and 0.027 , respectively; Fig. $3 B$ and $5 B$ ). Passive force and MTU stiffness in the $\mathrm{SP}$ group were not significantly different at $\mathrm{L}_{\mathrm{opt}}$. These results show that spasticity affected PL and GA muscles in a similar manner.

Length-force characteristics of SO. For passive and active length-force characteristics of SO (Fig. 3C), ANOVA indicated an MTU length effect ( $P=0.026$ and 0.001 , respectively) but no group effect. In contrast to GA and PL, no statistical interaction was found. Neither did maximal active force, slack length, or optimum length of the SP group differ from those of controls $(P=0.026$, Fig. $4 A$, Table 2$)$. However, passive MTU stiffness at $\mathrm{L}_{\mathrm{opt}}$ was higher in the SP group than in controls $(P=0.002$, Fig. $5 B)$. These results indicate that in contrast to GA and PL, active length-force characteristics of SO in the SP did not differ from those of controls, and passive MTU stiffness at $\mathrm{L}_{\mathrm{opt}}$ was increased in the SP group.

Epimuscular myofascial force transmission. Total force of TA was reduced in the SP group $(P<0.001)$, but was not
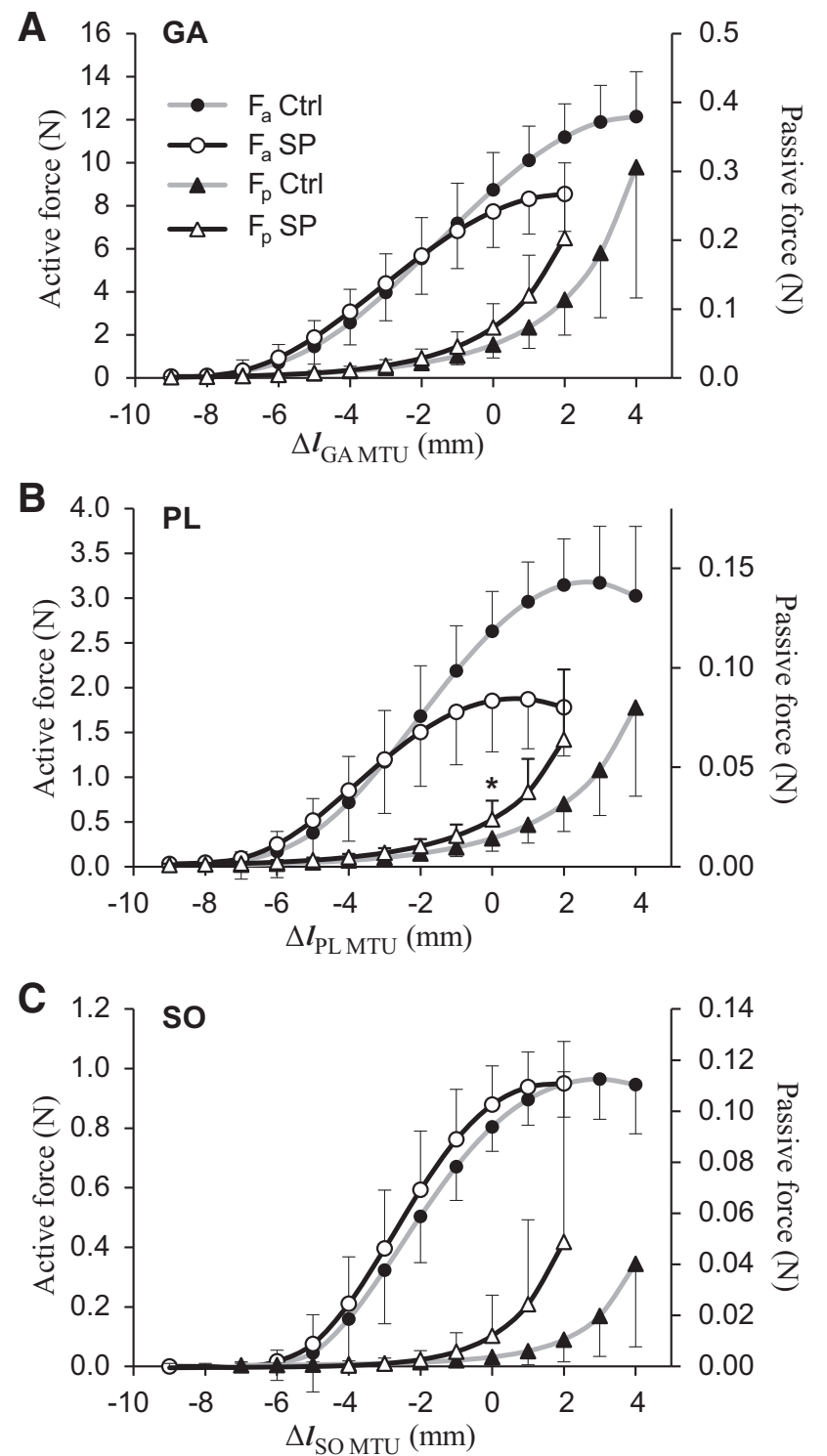

Fig. 3. Active and passive length-force curves of GA, PL, and SO. A: active and passive force of GA $\left(\mathrm{F}_{\mathrm{a}}\right.$ and $\mathrm{F}_{\mathrm{p}}$, respectively). Length effect $(P<0.001)$ and interaction (group $\times$ length, active $P=0.002$, passive $P=0.045$ ) were found, but there was no group effect. $B$ : active and passive force of PL. For active PL force, a length effect $(P<0.001)$ and interaction $(P<0.001)$ were found, but there was no group effect. For passive PL, group $(P=0.023)$ and length $(P<0.001)$ effects were found, as was interaction (group $\times$ length $P=0.028)$. The passive force at $\mathrm{L}_{\text {ref }}$ was higher in spastic rats than in control rats $(* P=0.026)$. The active force at $\mathrm{L}_{\text {ref }}$ was not affected by spasticity. $C$ : active and passive force of SO. Length effect was found (active $P=0.001$, passive $P=0.026$ ), but no group effect or interaction was found. Values are means $\pm \mathrm{SD}$. 
A Maximal active force

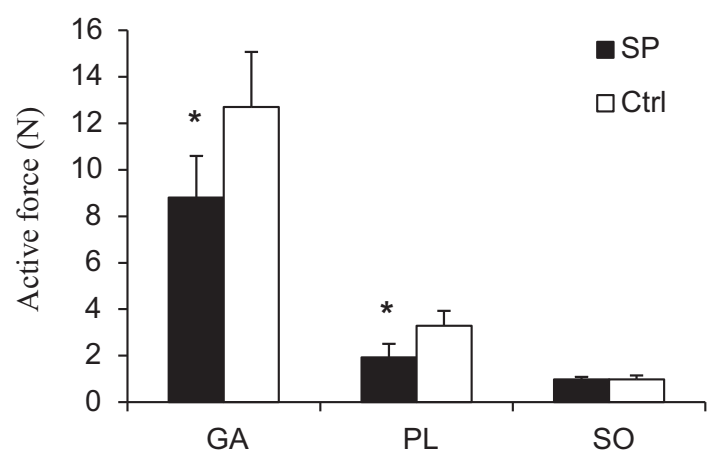

B Passive force at $L_{\text {opt }}$

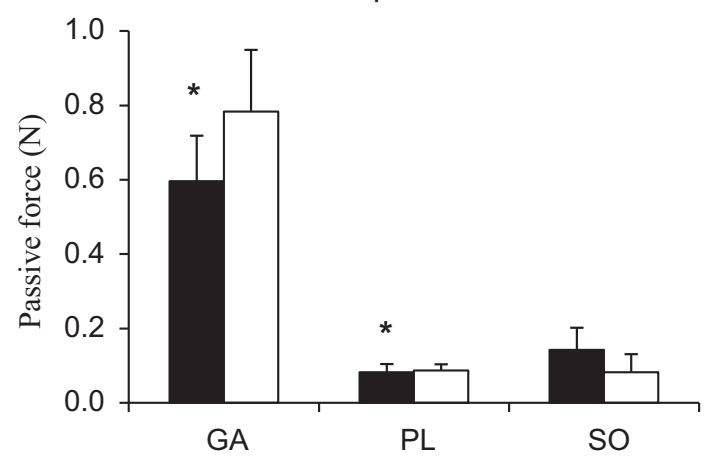

Fig. 4. Active $(A)$ and passive $(B)$ force at $\mathrm{L}_{\text {opt }}$. The active force at $\mathrm{L}_{\text {opt }}$ is the maximal active force per definition. ${ }^{*}$ Significant difference $(P<0.05)$. Values are means $\pm \mathrm{SD}$.

affected by changes in MTU length of the active calf muscles (protocol 1, Fig. 6A). Also, when force and length were normalized to total TA force at $\mathrm{L}_{\mathrm{ref}}$ and the length range between $\mathrm{L}_{\text {slack }}$ and $\mathrm{L}_{\text {opt }}$ (ascending length range), respectively, no group effect, MTU length effect, or interaction was found (Fig. 6B). These results indicate no mechanical interaction between the calf muscles and the antagonistic TA for both groups. However, mechanical interaction was found between GA and synergistic SO and PL muscles in both the spastic and control groups (protocol 2, Fig. 7). Increasing MTU length of GA distally decreased total force exerted at the distal tendons of SO and PL muscles (GA length effect $P<0.001$ ) despite constant MTU length of SO and PL. However, ANOVA indicated no significant interaction, indicating that the curves could not be statistically distinguished from being parallel to each other. In agreement with the length-force characteristics, no difference between groups was found for absolute SO force, but for absolute PL force, a group effect was found $(P=0.002$, Fig. 7A). On average across GA muscle lengths, SO force decreased by $45 \%$ in the SP group and by $44 \%$ in controls. PL force decreased by $19 \%$ in the SP group and by $20 \%$ in controls. Effects of MTU length of GA was also found when SO and PL forces were normalized to SO and PL forces at $\mathrm{L}_{\mathrm{ref}}$, respectively, and MTU length of GA was normalized to the length range between $\mathrm{L}_{\text {slack }}$ and $\mathrm{L}_{\text {opt }}$ of GA $(P<0.001$, Fig. $7 B)$. Normalization eliminated the group effect on PL force. These results suggest substantial mechanical interaction between GA and synergistic SO and PL muscles. However, the extent of synergistic and antagonist mechanical interaction was not affected by spasticity.

\section{DISCUSSION}

This is the first study describing length-force characteristics of skeletal muscles in a spastic mutant rat model. Spasticity affected the length-force characteristics for the whole group of calf muscles, and for the calf muscles individually. A main finding was that the effects of spasticity were muscle specific. Our first three hypotheses (narrower length-force curve and shift of length-force curve toward a more plantar-flexed position, reduced maximal active force, and increased passive stiffness) were confirmed for GA and PL muscles but not for SO. The extent of mechanical interaction was not affected by spasticity and thus, our fourth hypothesis was not confirmed.

Length-force characteristics of the calf muscles. The GA muscle was the dominant muscle of all calf muscles in the Han-Wistar rat. Hence the length-force characteristics of the entire group of calf muscles were similar to those of GA. Consistent with previous findings in human patients $(1,2,13)$, we found reduced maximal active forces of GA and PL in spastic rats. Loss of muscle volume has been reported in humans with spasticity $(4,31,34,35)$ and, therefore, atrophy is believed to be the main cause of muscle weakness. In the present study, maximal active force of GA was decreased by $31 \%$, whereas GA muscle mass was decreased by only $14 \%$, albeit not significantly. This suggests that part of the lower maximal active force should be explained by factors other than loss of muscle mass. Because the muscles were maximally excited, the most likely candidate is a decrease in specific tension.

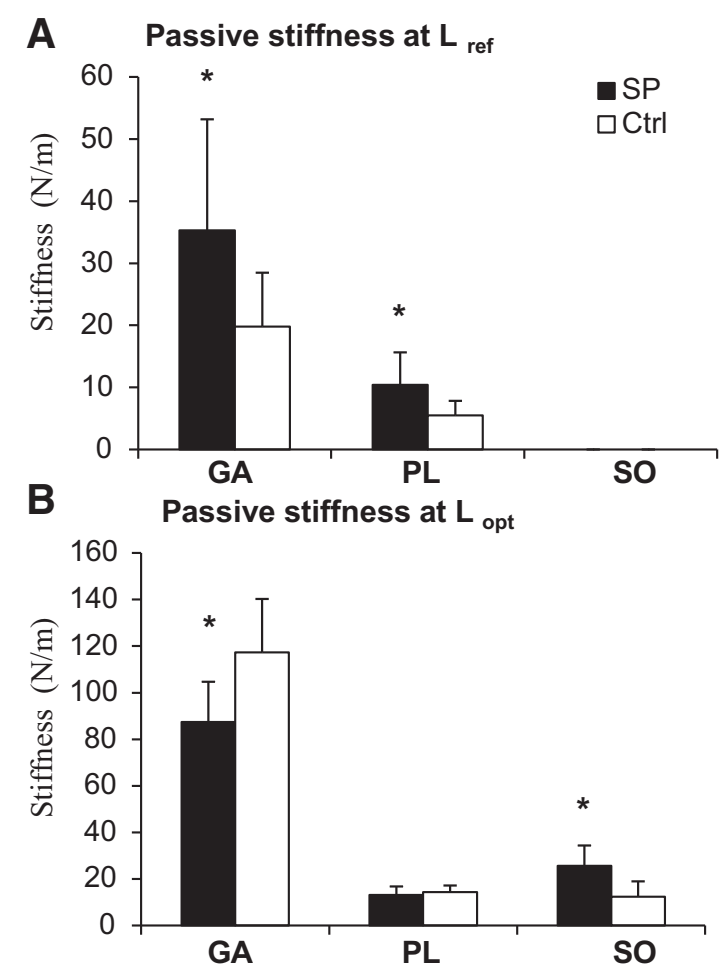

Fig. 5. Passive stiffness at $\mathrm{L}_{\mathrm{ref}}(A)$ and $\mathrm{L}_{\mathrm{opt}}(B)$. Passive stiffness of $\mathrm{SO}$ at $\mathrm{L}_{\mathrm{ref}}$ is not presented because ANOVA did not indicate group effect or interaction. $*$ Significant difference $(P<0.05)$. Values are means $\pm \mathrm{SD}$. 
A Absolute TA forces, protocol 1

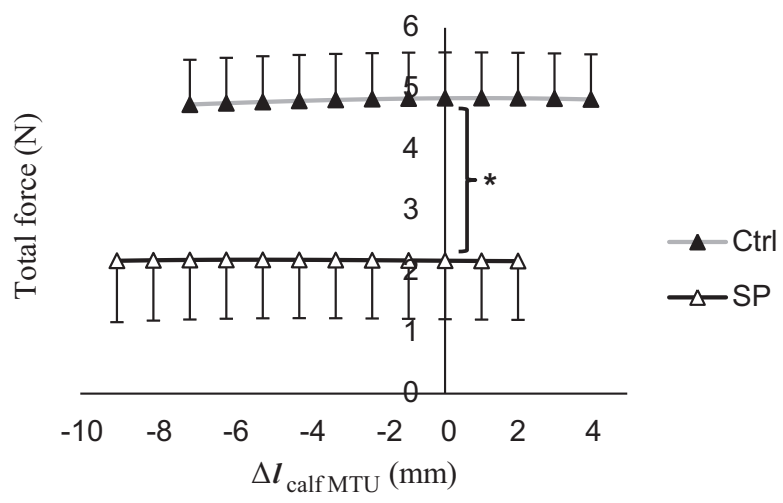

B Relative TA forces, protocol 1

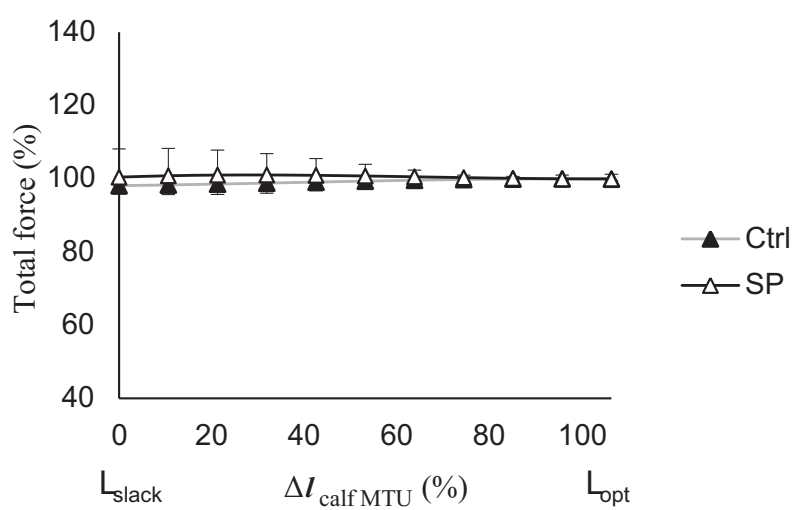

Fig. 6. Total force of TA in protocol 1. A: force is absolute total force of TA and length is relative to reference length $\left(\mathrm{L}_{\mathrm{ref}}=0 \mathrm{~mm}\right)$ of the calf muscles. A group effect was found $(* P<0.001)$, but no length effect or interaction was found. $B$ : force is normalized to the absolute total force of TA at $\mathrm{L}_{\mathrm{ref}}$. The length is normalized to the length range between active slack length and optimum length of the calf muscles. No significant effects or interaction were found. Values are means \pm SD.

Consistent with our hypothesis and previous indirect assessments in human studies $(2,3)$, passive force and stiffness of GA and PL were increased markedly at reference length $\left(90^{\circ}\right.$ ankle and knee angle) in the spastic rats. This indicates stiffer MTUs in the spastic rats because anatomical dimensions were unaffected by spasticity (Table 1). Passive stiffness was reduced by $26 \%$ in GA and $9 \%$ in PL at optimum length. This may be explained by shorter MTU length of GA and PL in the spastic rats at optimum length (see Table 2). For GA and PL, optimum length was lower with respect to reference length, and the active length-force curve was narrower in the spastic rats. The absolute optimum length of GA was also lower because the absolute MTU length of GA at reference length was the same in the two groups (Table 1). These findings are consistent with length-force characteristics on the basis of net ankle joint moments and ultrasound imaging of spastic GA (2, $15,16)$. The lower optimum length and narrower length-force curve can be explained by a number of factors: shorter muscle belly, fewer sarcomeres in series, stiffer and/or shorter tendon, or a decreased distribution of mean sarcomere length between fibers. We found no indications of shorter muscle belly or tendon length in the present study (Table 1). The number of sarcomeres in series and sarcomere length distribution were not assessed in the present study, but will be investigated in future studies using this spastic rat model. The steeper passive lengthforce curves suggest an increased stiffness of the MTU, but whether this is the result of a stiffer tendon or muscle belly cannot be distinguished in the present study.

The spastic rats were standing and walking with a more plantar-flexed position of the ankle joint (see Supplementary Material), which is consistent with the increased passive stiffness and lower optimum length of GA and PL. This could indicate that the muscles have adapted to being active at more plantar-flexed positions of the ankle. However, the plantarflexed ankle position could also be a result of spasticitymediated changes in muscle structure and mechanical properties. In summary, the length-force characteristics of GA and PL muscles are affected by spasticity, but additional research is required to clarify precise causes.

Muscle-specific effects of spasticity. In contrast to GA and $\mathrm{PL}$, and to our hypotheses, active length-force curves of SO were not affected by spasticity. Passive force and stiffness of SO at optimum length were increased in spastic rats, which was also different than the results of GA and PL muscles. SO

\section{A Absolute SO and PL forces, protocol 2}

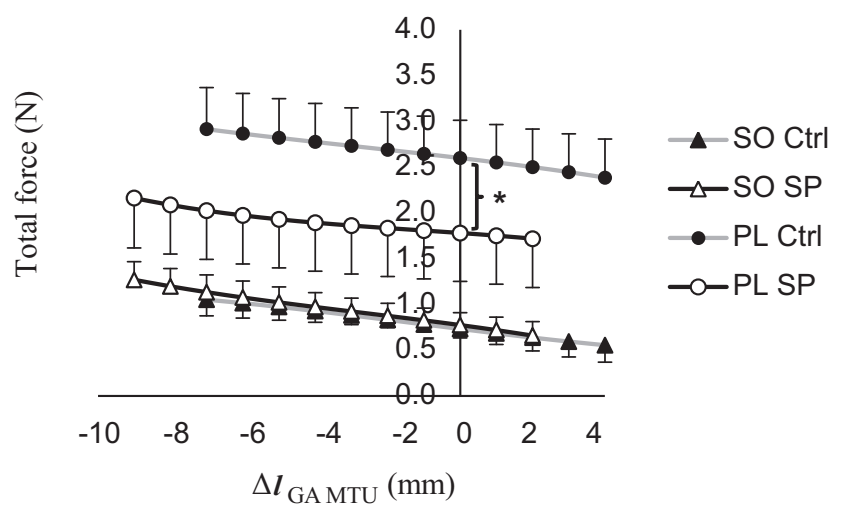

B Relative SO and PL forces, protocol 2

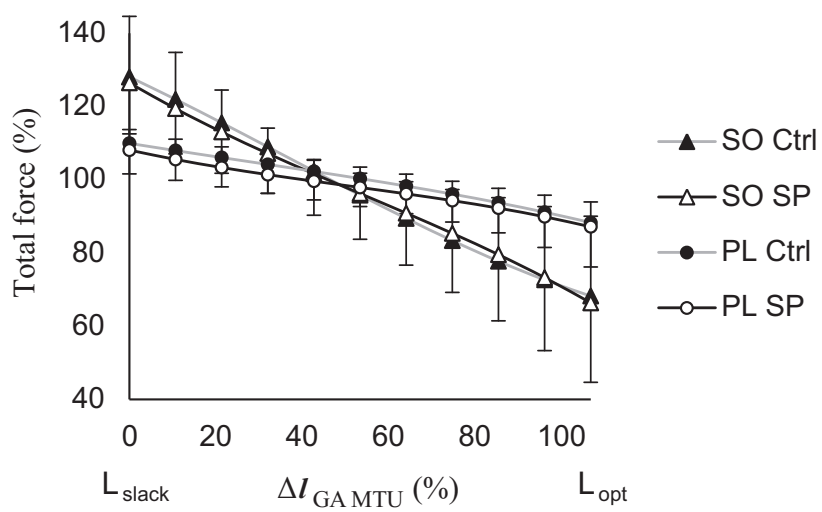

Fig. 7. Total force of PL and SO in protocol 2. A: force is absolute total force of PL and SO and length is relative to reference length $\left(\mathrm{L}_{\mathrm{ref}}=0 \mathrm{~mm}\right)$ of GA. Length effect for both PL and SO $(P<0.001)$ and group effect for PL were found $(* P=0.002)$, but there was no interaction. $B$ : force is normalized to the absolute total force of PL and SO, respectively, at $\mathrm{L}_{\text {ref. }}$. The length is normalized to the length range between active slack length and optimum length of GA. A length effect was found for both PL and SO $(P<0.001)$, but there was no group effect or interaction. Values are means \pm SD. 
muscle differs from GA and PL in rats with regard to muscle architecture [shallower pennation angle (14)], fiber type composition [more type I fibers (14)], and utilization [longer duration and more stabilizing (22)]. One study using biopsies from human spastic muscles has reported an increased passive tension in type II muscle fibers, but not in type I muscle fibers (36), which may contribute to muscle-specific effects of spasticity. In addition, SO is constantly active during low-intensity tasks such as standing and walking, whereas GA and PL are activated appreciably during tasks involving greater mechanical demands, such as running and jumping (22). GA, PL, and $\mathrm{SO}$ all have an antigravity function, and antigravity muscles have been reported to be the most affected by spasticity in humans (45). Our findings suggest that these antigravity muscles are not affected equally. The continuous activation of SO may protect the muscle from spasticity effects.

Because muscle spasticity in humans is diagnosed on the basis of whole limb properties, it is not known whether each of the individual calf muscles can be defined as spastic and whether muscles of the same compartment are equally affected by spasticity. Our results suggest that spasticity can affect muscles from the same compartment differently. It should be noted that the architecture of SO in rat $(14,23)$ does not resemble that of SO in humans $(18,25)$ and hence, comparisons should be made with caution. Nevertheless, our findings suggest that it would be of interest to investigate more than one muscle (typically GA) when investigating the mechanical properties of human spastic limbs.

Epimuscular myofascial force transmission. In contrast to our hypothesis, the extent of mechanical interaction between synergistic and antagonistic muscles was not affected by spasticity. Mechanical interaction depends on the mechanical properties of the epimuscular tissues. Therefore, this suggests that the altered limb mechanics in the spastic rats cannot be attributed to differences in epimuscular tissue mechanics, but that they originate from altered mechanics of individual muscular structures.

Evidence of epimuscular myofascial force transmission between GA and synergistic SO and PL was found, but not between the calf muscles and antagonistic TA. In contrast, epimuscular myofascial force transmission between antagonistic muscles has been observed in normally developed rats (21, $27,33,38,47)$. In those studies, epimuscular myofascial force transmission was observed between neighboring antagonistic muscles, or with TA at a relatively high length, whereas in our study the restrained TA muscle was kept at a rather low length (corresponding to a $90^{\circ}$ ankle angle). These different experimental conditions most likely explain the absence of antagonistic interaction in the present study. However, different experimental conditions are not expected to give rise to group differences, because we also did not find effects of spasticity on mechanical interactions between synergistic muscles.

The spastic rat model. The major advantage of using a rat model is that it allows for studying mechanical properties of several individual muscles, whole muscle compartments, and interaction between muscles and muscle compartments of the same spastic limb. Furthermore, both standardized limb positions (e.g., $90^{\circ}$ knee and ankle angle) and standardized muscular conditions (optimum length) can be compared. This has not been carried out before in a spastic animal model and will not be possible in human patients.
The model used in the present study is specifically phenotyped by limb spasticity, which resembles the typical symptoms in human patients suffering from muscle spasticity; that is, limb rigidity, plantar flexed ankles, crooked hands, and in general, impaired motor control (see Supplementary Material). Previously, this mutant rat model has been used for neural studies exclusively $(7,39,44)$. The mechanical properties of the spastic rat calf muscles found in the present study are consistent with the reduced functional range of motion and increased stiffness reported clinically in human limbs affected by spasticity $(17,31,32,40)$, and the narrower active lengthforce curves estimated in in vivo studies on human GA $(2,15$, 16). Thus the spastic rat model used in the present study mimics several important features of spasticity in human patients, and may be used to elucidate the mechanisms responsible for changes in muscle and limb mechanical properties observed in patients with spasticity.

Conclusions. GA and PL muscles were affected by spasticity in a similar way as reported for human patients with spastic cerebral palsy or stroke. That is, narrower active length-force curves, lower optimum length, and increased passive MTU stiffness at the same joint position. Length-force characteristics of the entire group of calf muscles were dominated by GA. Interestingly, the effects of spasticity were muscle specific within the posterior crural compartment. Because the extent of mechanical interaction between muscles was not affected by spasticity, we conclude that the altered limb mechanics in spastic rats originate from changes in individual muscular structures.

\section{ACKNOWLEDGMENTS}

We thank S. Peter Magnusson and Rene B. Svensson for comments on the manuscript.

\section{GRANTS}

Support for this study was provided by Division for Earth and Life Sciences of the Netherlands Organization for Scientific Research Grant 864-10-011 to H. Maas.

\section{DISCLOSURES}

No conflicts of interest, financial or otherwise, are declared by the author(s).

\section{AUTHOR CONTRIBUTIONS}

Author contributions: A.T.O., B.R.J., and H.M. conception and design of research; A.T.O. and G.C.B. performed experiments; A.T.O. analyzed data; A.T.O., B.R.J., and H.M. interpreted results of experiments; A.T.O. prepared figures; A.T.O. drafted manuscript; A.T.O., B.R.J., T.L.U., R.W.C., G.C.B., and H.M. edited and revised manuscript; A.T.O., B.R.J., T.L.U., R.W.C., G.C.B., and H.M. approved final version of manuscript.

\section{REFERENCES}

1. Bandholm T, Rose MH, Slok R, Sonne-Holm S, Jensen BR. Ankle torque steadiness is related to muscle activation variability and coactivation in children with cerebral palsy. Muscle Nerve 40: 402-410, 2009.

2. Barber L, Barrett R, Lichtwark G. Medial gastrocnemius muscle fascicle active torque-length and Achilles tendon properties in young adults with spastic cerebral palsy. J Biomech 45: 2526-2530, 2012.

3. Barber L, Barrett R, Lichtwark G. Passive muscle mechanical properties of the medial gastrocnemius in young adults with spastic cerebral palsy. J Biomech 44: 2496-2500, 2011.

4. Barber L, Hastings-Ison T, Baker R, Barrett R, Lichtwark G. Medial gastrocnemius muscle volume and fascicle length in children aged 2 to 5 years with cerebral palsy. Dev Med Child Neurol 53: 543-548, 2011. 
5. Booth CM, Cortina-Borja MJ, Theologis TN. Collagen accumulation in muscles of children with cerebral palsy and correlation with severity of spasticity. Dev Med Child Neurol 43: 314-320, 2001.

6. Botte MJ, Nickel VL, Akeson WH. Spasticity and contracture. Physiologic aspects of formation. Clin Orthop Relat Res Aug: 7-18, 1988.

7. Brunson KL, Khanna A, Cromwell HC, Cohen RW. Effect of the noncompetitive NMDA antagonists MK-801 and ketamine on the spastic Han-Wistar mutant: a rat model of excitotoxicity. Dev Neurosci 23: 31-40, 2001

8. Cohen RW, Fisher RS, Duong T, Handley VW, Campagnoni AT, Hull CD, Buchwald NA, Levine MS. Altered excitatory amino acid function and morphology of the cerebellum of the spastic Han-Wistar rat. Brain Res Mol Brain Res 11: 27-36, 1991.

9. Davy DT, Audu ML. A dynamic optimization technique for predicting muscle forces in the swing phase of gait. J Biomech 20: 187-201, 1987.

10. de Bruin M, Smeulders MJ, Kreulen M. Flexor carpi ulnaris tenotomy alone does not eliminate its contribution to wrist torque. Clin Biomech (Bristol, Avon) 26: 725-728, 2011.

11. de Bruin M, Smeulders MJ, Kreulen M. Why is joint range of motion limited in patients with cerebral palsy? J Hand Surg Eur Vol 38: 8-13, 2013

12. Dietz V, Sinkjaer T. Spastic movement disorder: impaired reflex function and altered muscle mechanics. Lancet Neurol 6: 725-733, 2007.

13. Elder GC, Kirk J, Stewart G, Cook K, Weir D, Marshall A, Leahey L. Contributing factors to muscle weakness in children with cerebral palsy. Dev Med Child Neurol 45: 542-550, 2003.

14. Eng CM, Smallwood LH, Rainiero MP, Lahey M, Ward SR, Lieber RL. Scaling of muscle architecture and fiber types in the rat hindlimb. $J$ Exp Biol 211: 2336-2345, 2008.

15. Gao F, Ren Y, Roth EJ, Harvey R, Zhang LQ. Effects of repeated ankle stretching on calf muscle-tendon and ankle biomechanical properties in stroke survivors. Clin Biomech (Bristol, Avon) 26: 516-522, 2011.

16. Gao F, Zhang LQ. Altered contractile properties of the gastrocnemius muscle poststroke. J Appl Physiol 105: 1802-1808, 2008.

17. Hägglund G, Wagner P. Spasticity of the gastrosoleus muscle is related to the development of reduced passive dorsiflexion of the ankle in children with cerebral palsy: a registry analysis of 2,796 examinations in 355 children. Acta Orthop 82: 744-748, 2011.

18. Hodgson JA, Finni T, Lai AM, Edgerton VR, Sinha S. Influence of structure on the tissue dynamics of the human soleus muscle observed in MRI studies during isometric contractions. J Morphol 267: 584-601, 2006.

19. Huijing PA, Baan GC. Extramuscular myofascial force transmission within the rat anterior tibial compartment: proximo-distal differences in muscle force. Acta Physiol Scand 173: 297-311, 2001.

20. Huijing PA, Baan GC, Rebel GT. Non-myotendinous force transmission in rat extensor digitorum longus muscle. J Exp Biol 201: 683-691, 1998.

21. Huijing PA, van de Langenberg RW, Meesters JJ, Baan GC. Extramuscular myofascial force transmission also occurs between synergistic muscles and antagonistic muscles. J Electromyogr Kinesiol 17: 680-689, 2007.

22. Hutchison DL, Roy RR, Hodgson JA, Edgerton VR. EMG amplitude relationships between the rat soleus and medial gastrocnemius during various motor tasks. Brain Res 502: 233-244, 1989.

23. Johnson WL, Jindrich DL, Roy RR, Reggie Edgerton V. A threedimensional model of the rat hindlimb: musculoskeletal geometry and muscle moment arms. J Biomech 41: 610-619, 2008.

24. Lance JW. The control of muscle tone, reflexes, and movement: Robert Wartenberg Lecture. Neurology 30: 1303-1313, 1980.

25. Lieber RL, Friden J. Functional and clinical significance of skeletal muscle architecture. Muscle Nerve 23: 1647-1666, 2000.

26. Maas H, Baan GC, Huijing PA. Intermuscular interaction via myofascial force transmission: effects of tibialis anterior and extensor hallucis longus length on force transmission from rat extensor digitorum longus muscle. $J$ Biomech 34: 927-940, 2001.

27. Maas H, Huijing PA. Synergistic and antagonistic interactions in the rat forelimb: acute effects of coactivation. J Appl Physiol 107: 1453-1462, 2009.
28. Maas H, Meijer HJ, Huijing PA. Intermuscular interaction between synergists in rat originates from both intermuscular and extramuscular myofascial force transmission. Cells Tissues Organs 181: 38-50, 2005.

29. Maas H, Sandercock TG. Force transmission between synergistic skeletal muscles through connective tissue linkages. J Biomed Biotechnol 2010: 575672, 2010.

30. Maas H, Yucesoy CA, Baan GC, Huijing PA. Implications of muscle relative position as a co-determinant of isometric muscle force: a review and some experimental results. J Mech Med Biol 3: 145-168, 2003.

31. Malaiya R, McNee AE, Fry NR, Eve LC, Gough M, Shortland AP. The morphology of the medial gastrocnemius in typically developing children and children with spastic hemiplegic cerebral palsy. $J$ Electromyogr Kinesiol 17: 657-663, 2007.

32. McDowell BC, Salazar-Torres JJ, Kerr C, Cosgrove AP. Passive range of motion in a population-based sample of children with spastic cerebral palsy who walk. Phys Occup Ther Pediatr 32: 139-150, 2012.

33. Meijer HJ, Rijkelijkhuizen JM, Huijing PA. Myofascial force transmission between antagonistic rat lower limb muscles: effects of single muscle or muscle group lengthening. J Electromyogr Kinesiol 17: 698707, 2007.

34. Mohagheghi AA, Khan T, Meadows TH, Giannikas K, Baltzopoulos V, Maganaris CN. Differences in gastrocnemius muscle architecture between the paretic and non-paretic legs in children with hemiplegic cerebral palsy. Clin Biomech (Bristol, Avon) 22: 718-724, 2007.

35. Mohagheghi AA, Khan T, Meadows TH, Giannikas K, Baltzopoulos $\mathbf{V}$, Maganaris CN. In vivo gastrocnemius muscle fascicle length in children with and without diplegic cerebral palsy. Dev Med Child Neurol 50: 44-50, 2008.

36. Olsson MC, Kruger M, Meyer LH, Ahnlund L, Gransberg L, Linke WA, Larsson L. Fibre type-specific increase in passive muscle tension in spinal cord-injured subjects with spasticity. J Physiol 577: 339-352, 2006.

37. Pittermann W, Sontag KH, Wand P, Rapp K, Deerberg F. Spontaneous occurrence of spastic paresis in Han-Wistar rats. Neurosci Lett 2: $45-49,1976$

38. Rijkelijkhuizen JM, Meijer HJ, Baan GC, Huijing PA. Myofascial force transmission also occurs between antagonistic muscles located within opposite compartments of the rat lower hind limb. J Electromyogr Kinesiol 17: 690-697, 2007

39. Schwarz M, Turski L, Janiszewski W, Sontag KH. Is the muscle relaxant effect of diazepam in spastic mutant rats mediated through GABA-independent benzodiazepine receptors? Neurosci Lett 36: 175180, 1983.

40. Sinkjaer T, Magnussen I. Passive, intrinsic and reflex-mediated stiffness in the ankle extensors of hemiparetic patients. Brain 117, Pt 2: 355-363, 1994.

41. Smeulders MJ, Kreulen M. Myofascial force transmission and tendon transfer for patients suffering from spastic paresis: a review and some new observations. J Electromyogr Kinesiol 17: 644-656, 2007.

42. Smeulders MJ, Kreulen M, Hage JJ, Huijing PA, van der Horst CM. Spastic muscle properties are affected by length changes of adjacent structures. Muscle Nerve 32: 208-215, 2005.

43. Smith LR, Lee KS, Ward SR, Chambers HG, Lieber RL. Hamstring contractures in children with spastic cerebral palsy result from a stiffer extracellular matrix and increased in vivo sarcomere length. $J$ Physiol 589: 2625-2639, 2011.

44. Uhlendorf TL, Van Kummer BH, Yaspelkis BB 3rd, Cohen RW. Neuroprotective effects of moderate aerobic exercise on the spastic HanWistar rat, a model of ataxia. Brain Res 1369: 216-222, 2011.

45. Welmer AK, Widen Holmqvist L, Sommerfeld DK. Location and severity of spasticity in the first $1-2$ weeks and at 3 and 18 months after stroke. Eur J Neurol 17: 720-725, 2010.

46. Woittiez RD, Baan GC, Huijing PA, Rozendal RH. Functional characteristics of the calf muscles of the rat. J Morphol 184: 375-387, 1985.

47. Yucesoy CA, Baan G, Huijing PA. Epimuscular myofascial force transmission occurs in the rat between the deep flexor muscles and their antagonistic muscles. J Electromyogr Kinesiol 20: 118-126, 2010.

48. Yucesoy CA, Huijing PA. Substantial effects of epimuscular myofascial force transmission on muscular mechanics have major implications on spastic muscle and remedial surgery. J Electromyogr Kinesiol 17: $664-$ 679, 2007. 